\title{
How nurse manager's position in the hospital hierarchy influences evidence-based practice implementation in nursing: A qualitative case study of the Nigerian acute care setting
}

\author{
Jude Ominyi*1, Chinwe Florence Samantha Ezeruigbo² \\ ${ }^{1}$ Faculty of Health \& Society, University of Northampton, United Kingdom \\ ${ }^{2}$ Department of Nursing Science, Ebonyi State University, Abakaliki, Ebonyi State, Nigeria
}

Received: January 2, 2019

DOI: $10.5430 /$ jnep.v9n6p14
Accepted: January 27, 2019

Online Published: January 30, 2019

URL: https://doi.org/10.5430/jnep.v9n6p14

\begin{abstract}
Background and objective: Evidence-based practice (EBP) is widely acknowledged as an essential aspect of healthcare delivery. Nurse managers are expected to contribute to the development of organisational cultures promoting EBP. However, there are indications that nurse managers are not necessarily empowered to drive implementation due to hierarchical constraints. This study explores how nurse manager's position in the hospital hierarchy influences EBP implementation in nursing, in the Nigerian acute care settings.

Methods: A qualitative case study methodology is utilised to gather data from two large acute care settings in Nigeria. Drawing on semi-structured interview, twenty-one ward managers and two nurse managers were interviewed. Data were transcribed and inductively analysed to generate four overarching themes.

Results: Nurse managers were hugely constrained by lack of autonomy to mobilise resources for EBP related activities. The hierarchical structure of the settings promoted top-down decision-making processes which in turn, limited nurse manager's visibility in the boardroom. Consequently, nurse managers were excluded from key strategic planning within the organisation and could not drive EBP implementation.

Conclusions: Findings highlight need for nurse managers to have greater visibility and managerial influence to enable them create opportunities for implementation of EBP in nursing. Implications for nursing management: Administratively, there is need for nurses to have greater involvement in management. Adequate authority and leadership visibility as well as managerial influence would enable nurse managers create opportunities for successful implementation.
\end{abstract}

Key Words: Evidence-based practice, Nursing practice, Nurse manager, Ward manager, Change in practice, Hospital hierarchy

\section{BACKGROUND}

Nursing practice is increasingly being subjected to public scrutiny due to perceived scandals in the healthcare industry. ${ }^{[1]}$ Thus, creating a context where nurses are required to justify the decisions they make with, and for patients. Owing to past mistakes, nurses now strive to utilise best available evidence to guide practice to meet expectations of service users. However, nurses' knowledge and skills is simply not

*Correspondence: Jude Ominyi; Email: Jude.Ominyi@northampton.ac.uk; Address: Faculty of Health \& Society, University of Northampton, UK, NN1 5PH, United Kingdom. 
enough $^{[2]}$ as the involvement of individual, teams as well as organisation is required to achieve successful implementation of evidence in practice. ${ }^{[3]}$ Several healthcare systems have in the past decade, acknowledged that evidence-based healthcare is essential, yet, there is a concern that what is known to be the best practice is not currently reflected in healthcare delivery. ${ }^{[3]}$

It is estimated that over $40 \%$ of patients do not receive care that is consistent with scientific evidence and over $20 \%$ of patients is at risk of being provided with potentially harmful care ${ }^{[4]}$ Therefore, there is a need to ensure delivery of EBP due to its crucial impact on the patient's well-being. ${ }^{[5]}$ Eighteen years ago, it is reported that it takes on average, seventeen years for research findings to become translated into practice. ${ }^{[6]}$ This slow pace of EBP implementation is due to complex multifaceted factors present within practice settings. ${ }^{[5,7,8]}$ Implementation scholars suggest that changes in current implementation policies and procedures create barriers that are beyond nurses' ability to tackle..$^{[1,9,10]}$

Indeed, available evidence shows that implementation of EBP in nursing largely depends on the practice setting. ${ }^{[7,9,11]}$ While suggesting that it may be better referred to as evidenceinformed practice, it is suggested that complexities surrounding EBP may have shifted from individual nurses to healthcare organisations. ${ }^{[9]}$ It goes without saying that there is no evidence indicating that nurses are unable to implement evidence, rather it is reported that nurse's ability to implement evidence relies on available support within the practice settings. ${ }^{[1]}$ Simply relying on nurses' knowledge and skills may not be deliver EBP, except if there is effective organisational drive for implementation processes. ${ }^{[12-14]}$ While several authors report impact of healthcare organisation on EBP in nursing practice, ${ }^{[9,15-17]}$ there exist confusion about the role of nurse managers, particularly in the Nigerian context.

The attitudes and actions of nurse managers may be part of the organisational factors influencing EBP implementation. Nurse managers are saddled with the responsibilities of facilitating EBP through provision of visionary leadership and support to staff nurses. ${ }^{[1,11]}$ Nurse managers are expected to contribute to the development of organisational cultures promoting EBP. ${ }^{[1]}$ However, there are indications that nurse managers do not necessarily have the prerogative to drive EBP due to hierarchical constraints within practice organisations. ${ }^{[18]}$ Nurse manager's ability to drive EBP has previously been linked to disempowerment as well as limited authority or support from organisational management. ${ }^{[18,19]}$ In the context of Nigerian healthcare system, the authority of nurse managers appears to be contingent on their positions

Published by Sciedu Press within the managerial hierarchy. This may be due to inherent bureaucracies enshrined in the management of public hospitals in the country, which entails that administrative positions are organically ascribed to individual.

Social structures within organisations can limit individuals' performances in their roles as resources are interlinked with agency to achieving one's role within the organisation. ${ }^{[20]}$ Therefore, the nurse manager's position within the hospital hierarchy may be limiting to EBP implementation since they may not have legitimate control over available resources. As organisational hierarchy denotes lines of authority for a given position, ${ }^{[18]}$ the nurse managers' position in the managerial hierarchy would likely impact on their leadership roles. While there is dearth of literature regarding the role of nurse managers in EBP implementation in Nigeria, there are indications that nurse managers may not have been accorded the authority to mobilise resources for EBP. In the light of the above, this study focuses on examining how nurse manager's positioning within the hospital hierarchy influences EBP implementation in clinical nursing.

\section{Aim}

This study explores how the nurse manager's position within the hospital hierarchy influences EBP implementation in nursing, in the Nigerian acute care setting.

\section{METHODS}

This study utilises a qualitative collective case study methodology. ${ }^{[21,22]}$ Qualitative case study focuses on exploring specific phenomenon while aiming to provide in depth interpretation of processes, actions, interactions relationships, and experiences occurring in a setting. ${ }^{[21]}$ Qualitative case study can be utilised to investigate and analyse single or collective (multiple) cases with the intention of capturing complex issues. ${ }^{[22]}$ It maintains outward looking and can collectively explore wider aspects of organisations, communities or societies. ${ }^{[22]}$ Qualitative case study provides opportunity for exploring successes and challenges facing implementation of healthcare interventions as it uncovers real life actions which corroborate told stories. ${ }^{[23]}$

\subsection{Design and settings}

A collective (multiple) case study design is utilised to enable generation of generate wider perspectives about the issue under inquiry. The medical and surgical units of two large hospitals (cases A and B) within one state of the country were selected for this study. These hospitals were relatively comparable in contextual characteristics as they both delivered acute care services to the public. These hospitals were selected using a combination of purposive and convenience 
sampling techniques. The intent was to explore EBP in a large, complex and challenging clinical environment where rich information was likely to be available. Hence, the use of purposive sampling technique. From a convenience perspective, the two hospitals were selected based on practicality, since they were in the same geographical areas which facilitated accessibility with minimal travelling. The authority of these hospitals, including nurse leaders were open and cooperative to this research.

\subsection{Selection of participants}

Participants include ward managers $(n=21)$ and nurse managers $(n=2)$ across the two hospitals. Participants were purposively selected based on their job titles and roles within the organisations. All participants were physically approached and recruited to participate in the study. None of the participants approached declined participation. Sample size in qualitative research is considered in relation to data saturation as well as depth of analysis rather than statistical representation. ${ }^{[24,25]}$ As qualitative research aims to explore individual's account of personal experiences about specific issues, ${ }^{[25]}$ increased sample may not necessarily warrant that new evidence will be uncovered if data saturation is achieved. ${ }^{[24]}$ Therefore, qualitative research sample is considered sizeable if it is enough to achieve data saturation and allow for in-depth analysis.

\subsection{Procedure for data collection}

In-depth semi-structured interviews were utilised to gather data from participants over a period of nine months between 2016 and 2017. This followed ethical approval by the Research Ethics Committees of the hospitals and subsequent provision of all relevant information about the study to potential participants. Informed consent was duly obtained from participants prior to interviews. Ward managers were first interviewed and then, the nurse managers. Interviews were conducted in English Language utilising interview guide which was specifically developed for this study. Interviews were face-to-face at the study sites and lasted for 50-90 minutes. Interviews were digitally audio-recorded and later transcribed verbatim. As there was dearth of literature about EBP in Nigeria, a very flexible and open approach that allowed participants freedom to share their perceptions, thoughts, feelings, and experiences was adopted. In doing this, each interview session was commenced by first introducing the study's focus, and then a presentation of one broad openended question which was pilot-tested with prompts. The interview was conducted by two researchers who have previously been trained in qualitative interviewing.

\subsection{Data analysis}

Cross-case, ${ }^{[22,26]}$ as well as thematic analysis approach were utilised in analysing study data. ${ }^{[24,27]}$ Data from each case were transcribed verbatim and analysed separately. First, data from case A was analysed to generate initial thematic codes which were used as frame of reference while analysing data from case B. Then, data from both cases were integrated as a single data set and collectively analysed to generate common themes. This cross-case analytic approach concentrated on establishing similarities and differences within and across the cases. This analysis was facilitated through NVivo 11, but was later completed manually. Initially, texts were labelled to generate codes, which were later revised as necessary. The coding process progressed from first to second level where codes were categorised in hierarchies. Essentially memos were used to capture insights as well as analytic thoughts during this process. Themes and patterns were searched for, while codes were constantly shuffled until no new categories were identifiable. Initial coding was undertaken by the first author while the second author reviewed and agreed after making relevant corrections.

\section{Findings}

Analysis of data as discussed in the section above yielded four overarching themes. These themes include resource control, top-down decision-making approach, lack of visibility in the boardroom and professional inequality. These themes are closely interrelated in their meanings and are individually discussed below.

\subsection{Theme 1: Resource control}

Findings show that control over available resources in both hospitals resources was not part of the nurse manager's roles. The right to allocate resources was retained in the hands of Medical Directors and other individuals occupying top hierarchy. The hierarchical structure existing in the settings entailed that nurse managers were generally positioned lower, and so, had limited authority to approve budgets for EBP related activities. As seen in the quotes below, nurse managers expressed that they have not been able to mobilise resources for EBP activities due to not having the authority over budgeting processes.

The medical director is the one who controls the hospital budget if he thinks it's important to remember us [nursing] when allocating resources then... like I said before the medical director is the person who has the power to allocate funds for evidence-based practice initiatives but in the meantime, he doesn't think it's important to fund EBP activities... so we can't really 
provide staff with relevant facilities otherwise... (Nurse manager, case A)

... I personally feel that our practice should be based on sound evidence but bulk of the problem that we [nursing] have a group is that we are placed in a position we don't directly manage the hospital budgets... it's unfortunate that we can't fund EBP because I don't have the power to make that budgetary decision. (Nurse manager, case B)

As seen in the quotes above, it is notable that nurse managers in both settings experienced hierarchical constraints due to the existing organisational structure. In the first quote above, nurse managers perceive that Medical Directors who were saddled with the responsibility of managing hospital resources did not consider it necessary to fund EBP activities. Therefore, funds were not provided for nurse managers to provide nurses with the required facilities to enable them initiate or institute EBP. In the second quote, nurse managers appeared to consider EBP relevant in nursing practice, however, they perceive that they lacked the authority to decide whether it was funded within the organisation. Consequently, initiation of EBP activities remained stifled due to lack of funding.

Further, nurse managers described how they proposed ideas that would have facilitated EBP to the Medical Director. However, these proposals did not get approved. This may imply that EBP was not considered a priority area to invest on by the Medical Director.

... even though I don't have the power to control the budget I have always argued for it... the management has to increase the nursing budget so we can at least sort out the most pressing issues... we need to recruit more nurses because so many of our nurses have left at least we can sort out the issue of time... staff have always said that they don't have time to think about evidence-based practice... we also need to fund some research projects but despite all the arguments, the Medical Director has the final say... (Nurse manager, case A)

As seen in the quote above, there are indications that nurse managers have desired to develop new ideas that would have facilitated EBP within the settings. Unfortunately, nurse managers could not do more than proposing ideas since they were not able to convince the management to provide budgetary provisions for implementation activities. There appear to be a conflict between managerialism and nursing professionalism as managerial-clinical relations seem to embody hegemony while preserving managerial rights in the command structure. There is an ideology that deprives nurse managers and nurses of their decision-making power while protecting the supremacy of managerial ranks. In a professional sense, nurses would have the autonomy to determine how nursing practice is delivered, however, top managers seem to be extremely in control of organisational goals, resources, decision-making and the way professional works were organised.

\subsection{Theme 2: Top-down decision-making approach}

Findings show that approaches to decision-making within the settings were largely top-down. Meanwhile, this topdown managerial approach practised in the settings appeared to have originated from the wider Nigerian healthcare context as findings suggest that the Ministry of Health imposed some initiatives on health professionals without necessarily considering outcomes of implementation. It was reported that bureaucrats in the Ministry of Health were developing clinical practice guidelines which they circulated across all health settings in the country. There were mandates requiring healthcare workers, including nurses to implement these clinical practice guidelines. As seen in the quotes below, this initiative was largely controlled by the bureaucrats in conjunction with the management of these hospitals.

...the ministry of health initially brought the idea of evidence-based practice and it was during a meeting that the Medical Director told us that minister has sent him a memo which says that we have to implement the national guideline... later on they brought copies of guidelines for us to implement... so we didn't have the chance to decide whether or not we can do it... (Ward manager, case A)

... as I said earlier when the ministry people brought guidelines and said we should implement them and the CMD too, I don't think they believe that we can contribute in the planning the guideline... the decide whatever they want and all we get are memos saying you have to do this and that... they don't care about how we do it. (Ward manager, case B)

As seen in the quotes above, it appears that there were initial negotiations between the Ministry of Health and hospitals' management on how to implement these national clinical practice guidelines. However, ward managers suggest that they were not invited to these negotiations and neither were 
they allowed the opportunity to make contributions on implementation processes. Non-consideration of nurses' inputs in implementation of the proposed new clinical practice guidelines may be due to nurses' lack of visibility at the upper management level.

\subsection{Theme 3: Lack of visibility in the boardroom}

Findings suggest that nurse managers lacked visibility at upper management level. Consequently, nurse managers' lack of voice and visibility in the boardroom deprived of them the authority to influence policy decisions. As seen in the quotes below, ward managers appear to suggest that they were willing to embark of projects that would foster EBP but they were given the opportunity.

It's all about the management they dictate to us what to do and as far as I'm concerned, we [nurses] are not permitted to bring up any new idea not to talk of carrying out a research project that will cost money... all that matters are that we obey their decisions... but the worse is that they don't even come down to ask how we can make things better if not for us, the patients... (Ward manager, case A)

... for me it's about who has the power to do what... the medical director and rest of them at the top are in charge, they are the ones that would approve whether we can adopt EBP or not, but that's the way the hospital is designed... you know how this thing work, you can't talk when you are not in the right position to do so... (Ward manager, case B)

From these quotes, nurse managers were not visible enough to create an atmosphere where nurses could freely make decisions on how practice may be changed. Apparently, there is sense of powerlessness among the nursing hierarchy and this might have explained why the nurse manager could not provide needed leadership that would have enabled nurses change practice. There are indications that low position of nurse managers might have sandwiched their ability to manoeuvre the authority.

However, ward managers seem to perceive that nurse managers have themselves contributed in complicating the adoption and development of evidence-based initiatives by nurses. Ward managers believe that nurse managers have focused more on maintaining their managerial positions regardless of their perceived lack of visibility to the upper management, thereby ignoring their clinical roles. There are indications that nurse managers were not bothered about whether nurses implemented EBP or not.
... she [nurse manager] is meant to be mentor us [nurses]... she is meant to supervise and provide opportunities where we can discuss clinical matters and not just remain there and think of budget and the rest... I understand that the management have put her in a tight position but I would expect my manager to identify with us and give us a listening ear. . how do we talk about standard of practice... how do we talk about evidencebased practice... many of the problems are not just management issues and I would expect my manager to be involved in developing skills for improved practice... (ward manager, case B)

\subsection{Theme 4: Professional inequality}

Findings show that differences in the hierarchy of various professional groups was problematic. Seemingly, clinical decision-making process was characterised by the traditional hierarchical nurse-doctor role relationship. The low position of nursing in the hierarchy of healthcare professional appears to have placed them in a situation where their roles were defined by physicians. As seen in the quotes below, nurses appear to suggest that they were constantly denied the opportunity to determine how they delivered nursing practices, even in cases where they felt they had the expertise to introduce relevant initiative for practice change.

... it's all about who does what... I mean I don't have the power to decide what needs to be changed or not, but the doctors do, because the power to decide belongs to the them and I have the feeling that some of them joined the profession so that they can dictate what happens... for me it's about hierarchy... (Ward manager, case A)

... I don't think any of us is ready to face them because as a profession we don't even have the power to do so... the way it is done here is that the doctors decide and we obey... it's either you obey instructions or back off. . . after all its about attending to patients in the best way you can even if it entails practising with our own bare knowledge alone... (Ward manager, case B)

As seen in the quotes above, there are indications that professional boundaries were constantly set and maintained, and may have resulted in nurse managers surrendering ownership of clinical decision-making authority. In case B, the nurse manager was strongly opposed by physicians when she advocated that the World Health Organisation's hand hygiene 
guidelines should be implemented following some adverse effects occurring in the unit.

Last year, I brought an idea that we should adhere to the World Health Organisation's hand hygiene checklist in our unit and I passed a circular that all ward managers should ensure this happened and we all complied, but I tell you, the doctors didn't want to comply simply because it's nurses' idea and it didn't hold... few months later, after we had Lassa fever incidence in the hospital with which two doctors and one nurse died and the checklist was brought up again by a doctor and it was implemented even the doctors because it was a doctor that brought it up this time. (Nurse manager, case B)

This may suggest that physicians were positioned to determine what form of evidence that were implemented. Although, the World Health Organisation's hand hygiene guidelines were later implemented, it was initially turned down when it was raised by nurse managers. This implies that evidence that was neglected when raised by a nurse manager was appreciated and subsequently implemented when it was the physician's idea. It appears that doctors did not adhere to available clinical practice guidelines except if it was presented by their medical colleagues. There are indications that nurse managers may have been socialised to remains subordinates in their roles which has impacted EBP implementation in the settings.

\section{Discussion}

The influence of hierarchy on the nurse manager's managerial autonomy was conspicuously revealed by findings of this study. Nurse managers in both settings lacked managerial autonomy required in driving implementation of new practices. There is no published literature in Nigeria related to impact of hierarchy on EBP implementation, however, there are studies from the international domain acknowledging these influences. ${ }^{[3,20]}$ In this study, structural power as well as hierarchy consistently appeared as key influencers of knowledge utilisation by nurses. Power differentials existing in the settings generated dominant-subordinate relationship between nurse managers and hospital managers (medical directors), leading to micro-political conflicts. Hybridity of roles complicated inter-professional relationships and deprived nurses of their autonomy. There were questions regarding nature of nursing professional autonomy and extent to which nurse managers could challenge the traditional power structure in this context as they seem to have normalised practices enforcing their subordination.
As seen in the findings of this study, nurse managers could not mobilise needed resources for effective change in practice innovations and were not visible at top management level to influence policy decisions. Nurse managers did not have access to highest level of power within the settings, and their perceptions reflected that of the wider societal discourses of nursing's invisibility and lack of voice in the Nigerian healthcare institution. Consequently, this had an impact on the delivery of evidence-based care. This was affirmed by nurse managers themselves when they expressed that they lacked managerial autonomy to mobilise or influence how resources were allocated within the settings. Nurse managers were not only downgraded but were marginalised in that they also expected nurses to initiate changes in practice initiatives as well as deliver evidence-based care to patients. It was not clear how nurses were expected to deliver EBP when they were not provided with relevant support in terms of making time for research activities, adequate staffing to reduce huge workloads and incentive for further training.

Nurse managers were hugely constrained by traditional budgetary procedures that consistently ignored nursing related projects. While junior nursing staff perceived that nursemanagers would have made necessary provisions for nursing practices, they were not visible enough to influence organisational decisions beyond the domain of nursing units. Lack of visibility of nurse managers and their exclusion from key decision-making as revealed in the findings implied that they were not influential in the organisation. It is noted that there is a risk as well as danger of uncertainty when those who are expected to show leadership are made to remain invisible. ${ }^{[27]}$ Again, this may relate to inherent bureaucracies in the Nigerian healthcare workforce in which healthcare institutions are traditionally structured in hierarchies. This may imply that directors of nursing at the Ministry of Health are not currently positioned to actively participate in healthcare decision-making at national level which again emphasises the issue of inclusion. It is unlikely that nurses at the hospital level will have access to required resources if their leaders are denied visibility or are restricted from participating or functioning at executive team level. It is argued that nurse leaders who are located further down the hierarchy of command are by far less likely to be influential; they are rather disempowered. ${ }^{[28]}$

Historically, nursing as a profession is associated with women as well as invisibility that is inherent in women's occupational group. ${ }^{[29]}$ Arguably, nursing (art of caring) as an occupational group was not until recently, seen as invisible commodity that did not contribute in building the economy. While acknowledging the interlink between leadership as well as power, there is an indication that the more nursing 
search for visibility, the more it becomes powerless. ${ }^{[30]}$ Indeed, nurse managers as well as nurse leaders must ensure collaboration with hospital managers to be able to contribute in policy decision-making. Nurses must not only assure credibility but also face reality of the present system by achieving visible positions that can enable them influence and mobilise resources required for implementing nursing best practices. Nurse leaders may be able to influence nursing care environment by becoming visible in the boardroom. ${ }^{[31]}$ This may be achievable if nurse leaders achieve the requisite competence to talk about financial and resource requirements. ${ }^{[31]}$

The crucial role of nursing leadership in EBP implementation is widely recognised, ${ }^{[18,32]}$ as it is believed that nursing visibility must extend beyond confines of nursing to policy as well as decision-making forums. ${ }^{[27]}$ Nurse managers cannot promote implementation activities if they do not have relevant managerial influence. ${ }^{[18]}$ Kueny $^{[19]}$ explored the role of nurse managers in EBP in nursing units of two hospitals in Canada. They found that workplace culture as well as structures influenced implementation of EBP in the settings. They reported that nurse managers who were empowered managed to drive change in practices as they could mobilise internal resources, support as well as quality assurance structures critical to EBP implementation. Previous studies reported that leadership, culture and structure were factors influencing knowledge utilisation in healthcare practice. ${ }^{[3,7]}$ These findings are consistent with that of this study as organisational cultures as well as structures created barriers preventing nurses from driving practice changes. Multiple factors characterising nursing work environment can have an impact on both nurses and clinical outcomes as nurse managers can neither drive change nor demonstrate accountability for nursing practice when they lack visibility. ${ }^{[33]}$ Manojlovich $^{[29]}$ found that nurses may be able to deliver better quality care and initiate activities capable of promoting positive work environment if they are empowered.

\section{Limitations and strengths of the study}

Findings of this study can be considered to have cast light on the influence of nurse manager's positioning on implementation of EBP in nursing in Nigeria. However, I must acknowledge that there are circumstances that may have limited the quality of evidence generated in this study. This is a report of a small qualitative study which is not intended to achieve generalisability in quantitative sense. Therefore, findings of this study may be judged in relation to the context of Nigerian acute care setting. Similar findings may be elicited from similar participants groups, characteristics, practices, behaviour patterns in similar settings. It may be a limitation that this study has only explored perspectives of ward managers as well as nurse managers, and have not necessarily involved patients as well as physicians other than hospital managers. Involving patients may not have necessarily changed the information as they are not experts in EBP implementation. Overall, this study makes significant contributions to a body of knowledge.

This is the first study in Nigeria exploring influence of nurse manager's positioning in the hospital hierarchy on EBP implementation. Previous studies investigating EBP in nursing in Nigeria have focused on individual determinants, utilising self-reported surveys as well as other quantitative approaches. ${ }^{[34-40]}$ These studies focused on nurses' perceptions of barriers and facilitators of EBP as well as research utilisation using a quantitative approach.

\section{IMPLICATIONS AND CONCLUSIONS}

Findings of this study suggest several practice and policy implications. This study's findings provide future direction for nursing practice, nursing administration as well as healthcare policies. Administratively, there is need for nurse managers to have greater involvement in management, particularly, regarding nursing practice. As seen in this study, nurse managers in these settings did not play active role in promoting the delivery of evidence-based changes. The situation may have been different if nurse managers were provided opportunities to manage or drive and initiate new ideas. There is need for nurse managers to have adequate authority and leadership visibility as well as managerial influence at all level of practice. It is imperative that nurse managers imbibed authoritative positions that would enable them to develop impetus to engage with knowledge mobilisation and dissemination. Passive positions as well as roles of nurse managers in these settings could not have allowed them opportunities for greater authority to drive practice changes. Based on findings of this study, nurse managers did not have rights to participate in both administrative as well as clinical decisionmaking.

Findings of this study reflects difficulties surrounding nursing practices. As notable in this study, patient safety in these settings was largely compromised. Currently, nurses are conflicted in how they desire to practise nursing and how they practised. There is imminent danger for patients or service users in this context and relevant stakeholders as well as healthcare managers must set aside economic priority in which they currently drive for. The government may wish to immediately restructure the healthcare workforce to minimise inherent obstructive structures. Traditionally, nursing practice is based on rituals, and does not seem to be meeting patient's healthcare needs. Nurses must be granted managerial prerogative as well as personal, collegial and professional 
autonomy to implement new ideas in the interest of patients. In this way, nurses may be able to achieve practice changes and provide nursing care in the way that is universally as well as professionally required.

\section{ACKNOWLEDGEMENTS}

I sincerely appreciate Dr Tracey Redwood, Dr Stuart Allen and Dr Evangelia Prokopiou for their support in proofreading this work while it was yet to be drafted as an article. Your immense contributions towards the completion of this study are notable. My sincere appreciation goes to the nurse managers and ward managers who participated in this study.

\section{CONFLicts of InTEREST Disclosure}

The authors declare that there is no conflict of interest.

\section{REFERENCES}

[1] Foxcroft DR, Cole N. Organisational infrastructures to promote evidence based nursing practice. Cochrane Library. 2005.

[2] Ellis P. Evidence-based practice in nursing. 3rd ed. London: SAGE; 2016.

[3] Seers K, Cox K, Crichton N, et al. FIRE (facilitating implementation of research evidence): a study protocol. Implementation Science. 2014; 7: 25. PMid:22453077 https://doi.org/10.1186/1748 $-5908-7-25$

[4] Graham ID, Logan J, Harrison MB, et al. Using the Ottawa Model of Research Use to implement a skin care program. Journal of Nursing Care Quality. 2006; 19: 18-24. https://doi.org/10.1097/0000 1786-200401000-00006

[5] Rycroft-Malone J. Promoting Action on Research Implementation (PARIHS). In: J., Rycroft Malone, \& T., Bucknall, T. (eds.) Models and Frameworks for Implementing Evidence-Based Practice: Linking Evidence to Action. Wiley-Blackwell; 2010a.

[6] Balas EA, Boren SA. Managing Clinical Knowledge for Healthcare Improvements. Germany: Schattauer; 2000

[7] Estabrooks CA, Squires JE, Hutchinson AM, et al. Assessment of variation in the alberta context tool: The contribution of unit level contextual factors and specialty in Canadian pediatric acute care settings. BMC Health Services Research. 2011; 11: 1-17.

[8] Melnyk BM, Gallagher-Ford L, Thomas BK, et al. A study of chief nurse executives indicates low prioritization of evidence-based practice and shortcomings in hospital performance metrics across the United States. Worldviews on Evidence-Based Nursing. 2016; 13(1): 6-14. PMid:26873371 https://doi .org/10.1111/wvn.12133

[9] Rycroft-Malone J. leadership and the use of evidence in practice. World Views on Evidence-Based Nursing. 2008a; 5(1): 1-2. PMid:18333922 https://doi.org/10.1111/j.1741-6787.20 $08.00111 . \mathrm{x}$

[10] Titler M, Cullen L, Griffin E, et al. Toolkit for promoting evidence based practice. Iowa City, IA: University of Iowa. 2007.

[11] Fink R, Thompson CJ, Bonnes D. Overcoming barriers and promoting the use of research in practice. JONA. 2005; 35(3): 121-129.

[12] Dopson S. A view from organizational studies. Nursing Research. 2007a; 56(4): S72-S77. PMid: 17625478

[13] Marchionni C, Ritchie J. Organizational factors that support the implementation of a nursing best practice guideline. Journal of Nursing Management. 2008; 16(3): 266-274. PMid:18324985 https: //doi.org/10.1111/j.1365-2834.2007.00775.x

[14] Wilson PM, Petticrew M, Calnan MW, et al. Disseminating research findings: what should researchers do? A systematic scoping review of conceptual frameworks. Implementation Science. 2010; 5: 91. PMid:21092164 https://doi.org/10.1186/1748-5908-5-91

Published by Sciedu Press
[15] Aita M, Richer M, Heon M. Illuminating the processes of knowledge transfer in nursing. Worldviews on Evidence-Based Nursing 2007; 4(3): 146-155. PMid:17850495 https://doi .org/10.111 $1 / j .1741-6787.2007 .00087 . x$

[16] Cummings GG, Estabrooks C, Midodzi WK, et al. Influence of organizational characteristics and context on research utilization. Nursing Research. 2007; 56: S24-39.

[17] Kitson A. What Influences the Use of Research in Clinical Practice? Nursing Research. 2007; 56(4): S1-S3.

[18] Wilkinson JE, Nutley SM, Davies HTO. An Exploration of the Roles of Nurse Managers in Evidence-Based Practice Implementation. Worldviews on Evidence-Based Nursing. 2011; 8(4): 236 PMid:21668735 https://doi .org/10.1111/j.1741-6787.20 $11.00225 . x$

[19] Kueny A, Shever LL, Mackin ML, et al. Facilitating the implementation of evidence-based practice through contextual support and nursing leadership. Journal of Healthcare Leadership. 2015; 7: 29-39. PMid:29355177 https://doi .org/10.2147/JHL.S45077

[20] Cheng L, Broome ME, Feng S, et al. Factors influencing implementation of evidence in Chinese nursing practice. Journal of Clinical Nursing. 2017; 26: 5103-5112.

[21] Stake RE. Qualitative case studies. In N. K.Denzin, \& Y. S.Lincoln (eds.), The Sage handbook of qualitative research. 3rd ed. Thousand Oaks: SAGE; 2005.

[22] Stake RE. The art of case study research. Thousand Oaks: SAGE; 1995.

[23] Simons H. Case Study Research: In-Depth Understanding in Context In Leavy, P. (eds). The Oxford Handbook of Qualitative Research. Oxford Handbooks Online; 2009.

[24] Pope C, Mays N. Qualitative Research in Health Care. 3rd ed. New Jersey: Blackwell; 2002.

[25] Pope C, van Royen P, Baker R. Qualitative methods in research on health policy. Qualitative Safety and Healthcare. 2006; 11: 148-152. https://doi.org/10.1136/qhc.11.2.148

[26] Yin RK. Research Methods and Designs. London: SAGE; 2014.

[27] Pedler N, Burgoyne J, Boydell T. A manager's Guide to Leadership. 2nd ed. England: McGraw Hill; 2010.

[28] Kanter RM. Men and Women of the Corporation. New York: Basic Books; 1977.

[29] Manojlovich M. Power and empowerment in nursing: looking backward to inform the future. Online Journal of Issues in Nursing. 2007; 12(1): 2 .

[30] Simpson R, Lewis P. An investigation of silence and a scrutiny of transparency: Re-examining gender in organization literature through the concepts of voice and visibility. Human Relations. 2005; 58(10): 1253-1275. https://doi.org/10.1177/0018726705058940

[31] Loman C. Corporate focus vital in the boardroom. 2010. 
[32] Newhouse R. Creating infrastructure supportive of evidence-based nursing practice: leadership strategies. Worldviews on Evidencebased Nursing. 2007; 4(1): 21-29. PMid:17355407 https://doi . org/10.1111/j.1741-6787.2007.00075.x

[33] Ramos A, Borrego-Ales Y. Empowering Employees: Structural Empowerment as Antecedent of Job Satisfaction in University Settings. Psychological Thought. 2014; 7(1): 28-36. https : //doi .org/10 .5964/psyct.v7i1.88

[34] Abimbola O. Conduct and utilization of research among nurses at federal capital territory, Abuja Nigeria. Journal of Nursing Education and Practice. 2014; 4(7): 139-147. https://doi.org/10.5430/ jnep.v4n7p139

[35] Adejumo PO, Guobadia PO. Nurses' attitudes to reading research articles and their perception of research utilization in clinical practice in a Nigerian City. Journal of Biomedical Sciences. 2013; 12(1): 46-56.

[36] Agbedia C, Okoronkwo I, Onokayeigho E, et al. Nurses' perspectives of the research practice gap in nursing. Open Journal of Nursing.
2014; 4: 95-100. https://doi.org/10.4236/ojn. 2014.42013

[37] Edet OB, Ella R, Esienumoh EE. Psychiatry nurses' knowledge-base, perceptions on research and research utilization in Calabar, Nigeria. International Professional Nursing Journal. 2011; 9(1): 112-119.

[38] Irinoye OO, Ayandiran EO, Fakunle I, et al. Nurses' perception $\&$ barriers to use of Information Communication Technology in a Teaching Hospital in Nigeria. Computer, Informatics, Nursing. 2013; 31(8): 394-400. PMid:23851710 https ://doi.org/10.1097/NX N. Ob013e3182997a6e

[39] Nwozichi C, Ojewole F. Perceived research utilization barriers among nurses in a rural hospital in Nigeria. International Journal of Advancements in Research and Technology. 2014; 3(1): 184-191.

[40] Ofi B, Sowunmi L, Dele E, et al. Professional nurses' opinion on research and research utilization for promoting quality nursing care in selected teaching hospitals in Nigeria. International Journal of Nursing Practice. 2008; 14: 243-255. PMid:18460066 https://doi.org/10.1111/j.1440-172X.2008.00684.x 